\title{
IL-6 induction of hepatocyte proliferation through the Tmub1-regulated gene pathway
}

\author{
MENGGANG LIU, HONGMING LIU, XIAOFENG WANG, PING CHEN and HONGXU CHEN \\ Department of Hepatobiliary Surgery, Daping Hospital, The Third Military Medical \\ University, Chongqing 400042, P.R. China
}

Received January 6, 2012; Accepted February 23, 2012

DOI: 10.3892/ijmm.2012.939

\begin{abstract}
The expression of transmembrane and ubiquitin-like domain containing 1 (Tmub1) is upregulated during liver regeneration, however, the function and underlying molecular mechanisms responsible for Tmub1 action remain to be determined. This study utilized BRL-3A rat liver cells for Tmub1 shRNA lentivirus infection and IL-6 stimulation. Semi-quantitative RT-PCR and western blot analysis were used to detect mRNA and protein expression levels, respectively. A $\left[{ }^{3} \mathrm{H}\right]$ thymidine incorporation assay was performed to assess changes in cell proliferation rates. Laser scanning confocal microscopy and immunoprecipitation-western blotting were used to assess the interaction between Tmub1 and calcium-modulating cyclophilin ligand (CAML) protein. The effect of Tmub1 on calcium ion influx into BRL-3A cells was measured by inverted fluorescence microscopy. The data showed that IL-6 treatment induced proliferation of rat hepatocytes and expression of Tmub1 mRNA and protein, while Tmub1 shRNA knocked down Tmub1 expression at both the mRNA and protein levels. Furthermore, compared to the negative control, Tmub1 shRNA-infected BRL-3A cells were highly proliferative with or without IL-6 stimulation. Tmub1 is colocalized with CAML in the hepatocellular cytoplasm, whereas knockdown of Tmub1 expression upregulated expression of CAML protein. Influx of $\mathrm{Ca}^{2+}$ into rat liver cells was also affected after Tmub1 knockdown. The data from the current study demonstrate that Tmub1 plays a negative role in IL-6-induced hepatocyte proliferation, and indicate that the interaction between Tmub1 and CAML may mediate the function of Tmub1 in hepatocytes.
\end{abstract}

\section{Introduction}

Tissue regeneration to replace damaged or lost cells plays an important role in maintaining homeostasis in the human body. Although different cells have different capabilities of regen-

Correspondence to: Dr Ping Chen, Department of Hepatobiliary Surgery, Daping Hospital, The Third Military Medical University, 10 Changjiangzhilu Daping, Chongqing 400042, P.R. China

E-mail: chenping10701@hotmail.com

Key words: transmembrane and ubiquitin-like domain containing 1, calcium-modulating cyclophilin ligand, IL-6, liver regeneration, hepatocyte proliferation eration or division, liver cells usually have strong regeneration abilities after liver injury or damage; hepatocytes rapidly proliferate to replace the damaged or lost cells. Nevertheless, cell regeneration is an extremely complex process that requires tight regulation of genes at both the transcriptional and translational levels. The resulting newly synthesized proteins interact with each other to promote the proliferation of adequate numbers of cells. Thus, investigation into the molecular regulation and mechanisms underlying hepatocellular proliferation will increase our understanding of the physiological processes of carcinogenesis.

Transmembrane and ubiquitin-like domain containing 1 (Tmub1) protein, also called hepatocyte odd protein shuttling (Hops), was first discovered by Della Fazia et al (1) through analysis of a hepatocellular cDNA library from a partial rat hepatectomy. The data showed that Tmub1 expression was nearly undetectable in normal liver tissues, but significantly increased after partial rat hepatectomy with peak levels occuring $48 \mathrm{~h}$ post-operation. Tmub1 protein localized to both the nuclei and cytoplasm of regenerating hepatocytes after the partial rat hepatectomy. Another study revealed that overexpression of Tmub1 inhibited proliferation of hepatoma H-35 and NIH-3T3 cells in vitro. At the molecular level, Tmubl interacts with eukaryotic elongation factor-1A (eEF-1A) to affect protein synthesis, which could be the potential mechanism by which cell proliferation is regulated (1). These findings contradict each other in regards to the function of Tmub1 in hepatocyte regeneration.

In our previous study, we demonstrated the upregulation of Tmub1 expression after partial rat hepatectomy, supporting a role for Tmub1 in the regulation of liver regeneration. However, it is unclear whether Tmub1 functions as a stimulator or inhibitor of cell proliferation, or whether Tmub1 affects the proliferation of normal hepatocytes. At the molecular level, Tmub1, as a shuttling protein, may interact with different molecules to regulate the regenerative processes in the liver. For example, IL-6 secreted by sinusoidal endothelial cells, was shown to play an important role in liver regeneration after partial hepatectomy (2). Treatment with IL-6 induces cell proliferation, although it is not clear whether IL-6 interacts with Tmub1 during hepatocyte proliferation. In addition, calcium-modulating cyclophilin ligand (CAML), first discovered in 1994, is ubiquitous in several tissues, and plays a role in the regulation of cell proliferation and apoptosis (3-9). CAML is able to regulate $\mathrm{Ca}^{2+}$ influx into cells $(3,5)$. In addition, CAML can interact with the epidermal growth 
factor receptor (EGFR) to control recycling of EGFR from the cytoplasm to the cell membrane, where it can then affect downstream signaling (6). The importance of $\mathrm{Ca}^{2+}$ in cell division is well known, and a previous study showed that cytosolic $\mathrm{Ca}^{2+}$ signals enhanced proliferation of hepatocytes during liver regeneration (10). EGFR is the target of transforming growth factor- $\alpha$ (TGF- $\alpha$ ), and proliferation induced by EGFR is indispensable in liver regeneration. CAML can also modulate the function of spindles to affect mitotic states (9). CAML regulates locomotor activity and wakefulness in mice by interacting with Tmub1 in brain tissue (11). Based on these findings, we hypothesized that the participation of Tmubl in hepatocyte regeneration (proliferation) occurs via Tmubl interaction with CAML protein. We first determined if IL- 6 regulation of hepatocyte proliferation is through Tmubl expression. We then knocked down Tmub1 expression to assess changes in phenotypes of rat hepatocytes, intracellular $\mathrm{Ca}^{2+}$ levels, and gene expression. In addition, we investigated the cellular localization of Tmub1, and interaction of Tmub1 with CAML.

\section{Materials and methods}

Cell culture and treatment. Rat liver BRL-3A cells were obtained from the Cell Bank of Academia Sinica (Shanghai, China), and cultured in Dulbecco's modified Eagle's medium (DMEM) supplemented with $10 \%$ fetal calf serum at $37^{\circ} \mathrm{C}$ in $5 \%(\mathrm{v} / \mathrm{v}) \mathrm{CO}_{2}$ (all from Invitrogen, Carlsbad, CA, USA). For IL-6 treatment, the optimal induction concentration and treatment time were derived from a previous study (12). Briefly, BRL-3A cells were starved with fetal calf serum-free DMEM for $2 \mathrm{~h}$, and rat recombinant IL- 6 was added to the cell culture at various concentrations $(0-30 \mathrm{ng} / \mathrm{ml})$ for different periods of time (0-24 h). Changes in cell proliferation were determined using the thymidine incorporation assay.

$\left[{ }^{3} \mathrm{H}\right]$ thymidine incorporation assay. Cells were cultured in 24-well plates until they reached the exponential growth phase. $\mathrm{H}^{3}$-TdR $(0.5 \mu \mathrm{Ci} / \mathrm{ml})$ was then added to the culture medium after the cells reached a density of approximately $50 \%$. The cells were incubated with $\mathrm{H}^{3}$-TdR for $6 \mathrm{~h}$, and then washed three times with phosphate-buffered saline (PBS) and detached with $0.25 \%$ trypsin. Cells were transferred to a filter membrane and incubated with $10 \%$ trichloroacetic acid for $1 \mathrm{~min}$. The filter membrane was then washed with dehydrated alcohol, desiccated at $80^{\circ} \mathrm{C}$ for $30 \mathrm{~min}$, and transferred into scintillation vials. After adding $5 \mathrm{ml}$ scintillation fluid, scintillation vials were placed in the dark for $2 \mathrm{~h}$, and cpm values were determined using a liquid scintillation counter. The mean $( \pm \mathrm{SE})$ of six experiments was calculated.

Construction and infection of Tmubl shRNA lentiviral vector. To knockdown Tmub1 expression, a lentivirus vector expressing Tmub1 shRNA was used. Briefly, four different targeting shRNA sequences against the rat Tmub1 gene (NM_198781.2) were designed and synthesized by Shanghai GenePharma Co., Ltd. (Shanghai, China). After annealing into double-strand DNA, these oligonucleotides were inserted into the PLL3.7 lentivirus expression vector between XhoI and $\mathrm{Hpa}$ sites. After amplification and sequence confirmation, these lentivirus vectors were packed and purified with lentiviral packaging systems (Tronolab, Lausanne, Switzerland). The interference effect of Tmub1 shRNA vectors was determined using hepatocellular infection experiments. Specifically, BRL-3A cells were cultured overnight and then infected with Tmub1 shRNA lentivirus vectors with a multiplicity of infection (MOI) of 50-100. Then, we chose an MOI of 70 to infect BRL-3A cells for 14 days, and cells with knockdown of Tmub1 expression were sorted with a flow cytometer. Tmub1 expression in BRL-3A cells was analyzed using semi-quantitative RT-PCR and western blotting techniques. The negative control cells were transfected with control vector.

RNA isolation and semi-quantitative reverse transcription polymerase chain reaction (RT-PCR). Total-RNA from cells was extracted with a TRIzol RNA extracting solution (Takara, Dalian, China). cDNA was synthesized using a reverse transcription kit (Takara) at $42^{\circ} \mathrm{C}$ for $60 \mathrm{~min}$, and $70^{\circ} \mathrm{C}$ for $5 \mathrm{~min}$. Tmub1 primers were 5'-TCTGTCGGAGAAACTTAGGA-3' (forward) and 5'-TCTGGAGGTGTTGATGCTG-3' (reverse). $\beta$-actin primers were 5'-GTGGGTATGGGTCAGAAGGA-3' (forward) and 5'-AGCGCGTAACCCTCATAGAT-3' (reverse). PCR cycles were programmed as follows: denaturation for $2 \mathrm{~min}$ at $94^{\circ} \mathrm{C}$, $20-25$ cycles of $30 \mathrm{sec}$ at $95^{\circ} \mathrm{C}, 30 \mathrm{sec}$ at $62^{\circ} \mathrm{C}$, and $30 \mathrm{sec}$ at $72^{\circ} \mathrm{C}$, and a final extension at $72^{\circ} \mathrm{C}$ for $10 \mathrm{~min}$. After testing different PCR cycles, we decided to use 25 cycles to amplify expression of these genes, because levels of all target genes were increased in the logarithmic phase. Each PCR product was measured using Hoechst Dye 33258 and a Hoefer TKO100 fluorometer. Electrophoresis strips were obtained with the gelatin imaging system and analyzed with the Quantity One software. Relative Tmub1 mRNA expression levels were normalized to $\beta$-actin.

Protein extraction and western blot analysis. To extract protein from cells, cells were washed with PBS, placed on ice and scraped into Eppendorf tubes. RIPA buffer was added to lyse the cells. After a $10 \mathrm{~min}$ incubation on ice, cells were centrifuged at 18,000 rpm for $45 \mathrm{~min}$, and the supernatant was collected in new tubes. The protein concentration was measured using Coomassie brilliant blue staining methods. Proteins were separated by SDS-polyacrylamide gel electrophoresis and transferred to polyvinylidene fluoride membranes. The membranes were blocked in 5\% milk for $1 \mathrm{~h}$, and incubated with primary antibody overnight at $4^{\circ} \mathrm{C}$. The next day, the membranes were washed three times with Tris-buffered saline-Tween-20 solution (TBST), followed by incubation with a secondary antibody for $1 \mathrm{~h}$ at room temperature. After three washes with TBST, membranes were visualized using enhanced chemiluminescence from Thermo Fisher Scientific (Shanghai, China), and band intensities were analyzed using the Quantity One software. Protein levels were normalized to the $\beta$-actin expression.

Laser scanning confocal microscopy. Cells were cultured on slides until a density of approximately $50 \%$, then washed with PBS and fixed with $4 \%$ paraformaldehyde for $15 \mathrm{~min}$. Slides were treated with $0.5 \%$ Triton X-100 after several washes with PBS, and then incubated in $1 \%$ bovine serum albumin in PBS for $30 \mathrm{~min}$ at room temperature, followed by incubation with anti-Tmubl and anti-CAML antibodies overnight at $4^{\circ} \mathrm{C}$. The next day, the slides were washed with PBS three times, and incu- 
$\mathbf{A}$

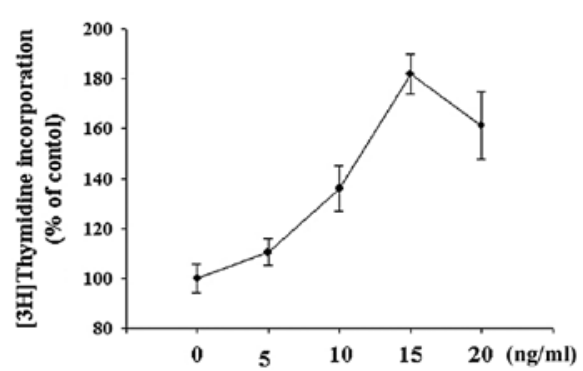

B

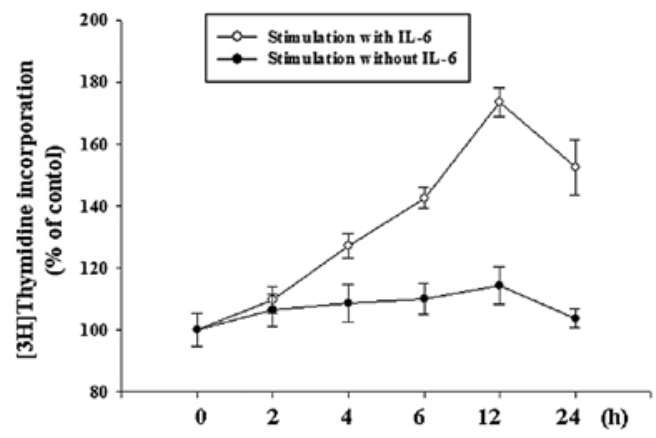

Figure 1. Effect of IL-6 on regulation of rat liver cell proliferation. The $\left[{ }^{3} \mathrm{H}\right]$ thymidine incorporation assay was used to detect changes in cell proliferation rates after treatment of BRL-3A cells with (A) recombinant rat IL-6 at various concentrations for (B) different periods of time.

$\mathbf{A}$
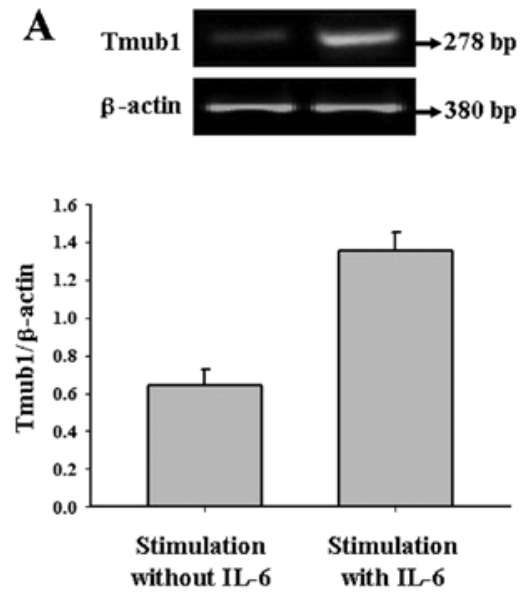

B
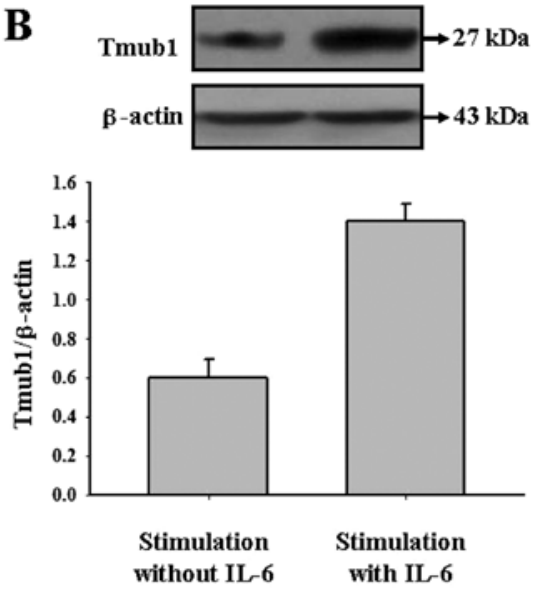

Figure 2. Effect of IL-6 on induction of Tmub1 expression. Rat liver BRL-3A cells were grown and treated with $15 \mathrm{ng} / \mathrm{ml} \mathrm{IL}-6$ for $12 \mathrm{~h}$. RNA and protein were extracted and subjected to (A) semi-quantitative RT-PCR and (B) western blotting analyses of Tmub1 expression. Experiments were performed in triplicate and repeated once.

bated with a donkey anti-goat secondary antibody for Tmub1 recognition and a goat anti-rabbit fluorescence secondary antibody for CAML recognition, for $1 \mathrm{~h}$ in the dark at room temperature. Slides were washed with PBS and counterstained with 4,6-diamidino-2-phenylindole, then mounted with a $50 \%$ glycerol solution. Cells were imaged under a laser confocal microscope, and staining results were scored as positive or negative after at least 20 cells were counted in a low-power field.

Immunoprecipitation and western blotting assay. Total protein was extracted and quantified using the aforementioned methods. Then each sample containing $300 \mu \mathrm{g}$ protein was incubated with $5 \mu \mathrm{g}$ anti-CAML antibody overnight at $4^{\circ} \mathrm{C}$ on a shaking table, and the next day was further incubated for $2 \mathrm{~h}$ with $15 \mu 1$ protein G-Sepharose. The immunoprecipitates were briefly centrifuged at $1000 \mathrm{rpm}$, and the cell pellet was washed three times with RIPA buffer. After samples were boiled for 5 min in 2X Laemmli sample buffer, immunoprecipitates were assessed by western blot analysis using an anti-Tmub1 antibody.

Measurement of $\left[\mathrm{Ca}^{2+}\right]$ i levels. Cells were grown and detached with $0.25 \%$ trypsin into a cell suspension, washed three times with D-Hank's solution, and incubated with $2 \mu \mathrm{M}$ Fura-2/AM for $30 \mathrm{~min}$ at $37^{\circ} \mathrm{C}$ in a $5 \%(\mathrm{v} / \mathrm{v}) \mathrm{CO}_{2}$ incubator. Then, cells were centrifuged to discard Fura-2/AM-containing DMEM, and washed three times with $37^{\circ} \mathrm{C}$ D-Hank's solution before being transferred into a new culture dish especially designed for inverted fluorescence microscopy. The cells were then viewed under a fluorescence microscope (Nikon Eclipse Ti, Tokyo, Japan); the excitation wavelength alternated between 340 and $380 \mathrm{~nm}$. Images were obtained from five randomly selected fields, and at least 10 cells were observed per visual field. The results were analyzed using Nis-Elements software (Tokyo, Japan), and presented as mean \pm SD.

Statistical analysis. Data were analyzed with the SPSS software, version 13.0 (Chicago, IL), and results are presented as mean \pm SD. The Least-Significant Difference test was used to determine differences among these independent subgroups. A $\mathrm{P}$-value $<0.05$ was considered to be statistically significant.

\section{Results}

Effect of IL-6 on the regulation of liver cell growth and Tmubl expression. In this study, we first assessed the effects of IL-6 on the regulation of liver cell growth by treating rat liver BRL-3A cells with different doses of IL-6 for various periods of time. We found that the most effective concentration and treatment time of IL- 6 on proliferation of rat hepatocytes was $15 \mathrm{ng} / \mathrm{ml}$ for $12 \mathrm{~h}$, respectively (Fig. 1). Therefore, we chose $15 \mathrm{ng} / \mathrm{ml}$ and a 12-h treatment for the rest of the experiments. 

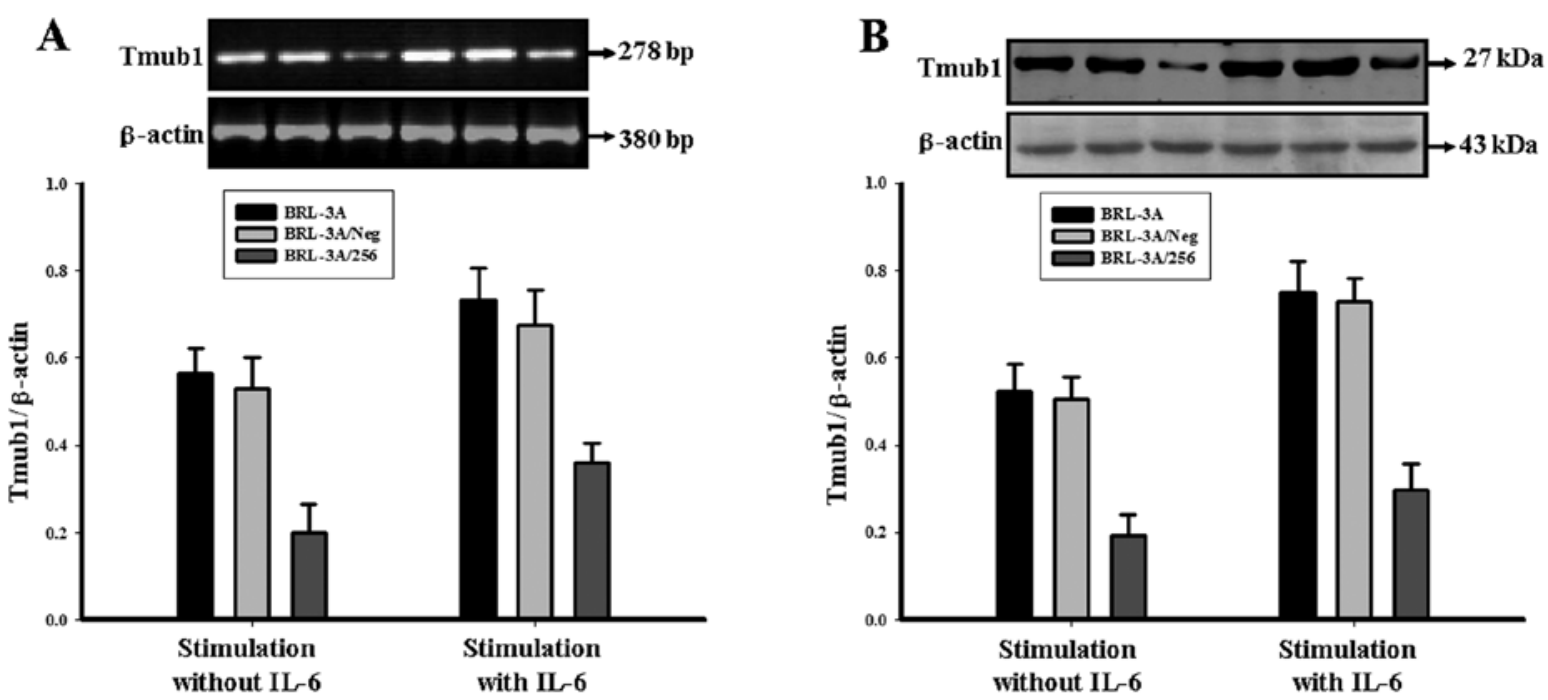

Figure 3. Knockdown of Tmub1 expression using Tmub1 shRNA in rat liver cells. Rat liver BRL-3A cells were stably infected with Tmub1 shRNA or the negative control lentivirus, respectively to generate stable Tmub1 knockdown cells (BRL-3A/256) and negative control cells (BRL-3A/Neg). The parental and stable cells were then grown and treated with or without $15 \mathrm{ng} / \mathrm{ml} \mathrm{IL}-6$ for $12 \mathrm{~h}$ and subjected to (A) semi-quantitative RT-PCR and (B) western blotting analyses of Tmub1 expression. Levels of Tmub1 mRNA and protein in BRL-3A/256 cells were lower than that in the negative control cells $(\mathrm{P}<0.05)$, but there were no differences found between BRL-3A and BRL-3A/Neg cells $(\mathrm{P}>0.05)$. In contrast, Tmub1 expression was upregulated in BRL-3A and BRL-3A/Neg cells with IL-6 treatment $(\mathrm{P}<0.05)$. Tmub1 expression in BRL-3A/256 was also induced with IL-6 treatment, but was lower than that in the negative control cells $(\mathrm{P}<0.05)$. The data are summarized from three independent experiments.

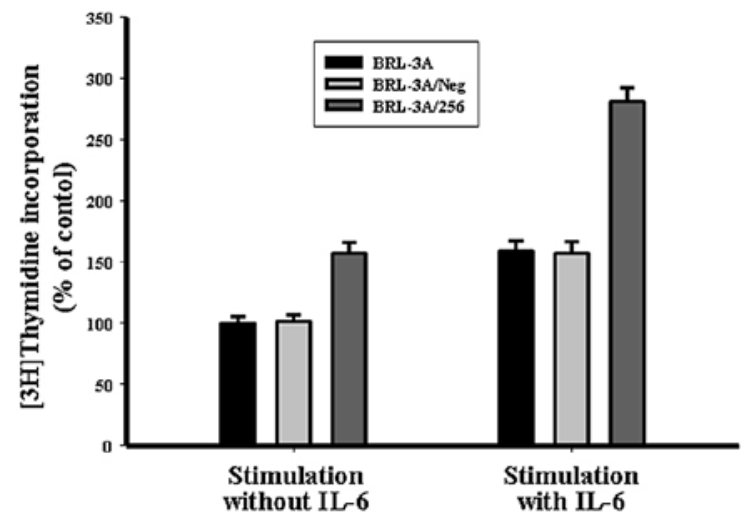

Figure 4. Effect of Tmub1 knockdown on the regulation of rat liver cell proliferation. Parental, negative control, and Tmub1 knockdown cells were grown and treated with or without $15 \mathrm{ng} / \mathrm{ml}$ for $12 \mathrm{~h}$, and then subjected to a $\left[{ }^{3} \mathrm{H}\right]$ thymidine incorporation assay. The data showed that $\left[{ }^{3} \mathrm{H}\right]$ thymidine incorporation efficiency in BRL-3A/256 cells was higher than in parental and negative control cells $(\mathrm{P}<0.05)$. The level of $\left[{ }^{3} \mathrm{H}\right]$ thymidine incorporation was increased after IL-6 treatment in all cells compared to no treatment $(\mathrm{P}<0.05)$; however, Tmub1 knockdown synergized the IL- 6 effects on cell proliferation $(\mathrm{P}<0.01)$. There were no differences between BRL-3A and BRL-3A/Neg cells with or without IL- 6 stimulation. The data are summarized from three independent experiments.

The effects were determined by measuring maximal $\left[{ }^{3} \mathrm{H}\right]$ thymidine incorporation into the genomic DNA of the cells.

Meanwhile, we analyzed Tmub1 expression in these IL-6-treated cells, and found that levels of Tmub1 expression significantly increased after treatment of hepatocytes with IL-6 (P<0.05) (Fig. 2). These data suggest that IL-6 was able to induce Tmub1 expression and rat hepatocyte growth.

Knockdown of Tmubl expression using a lentiviral vector carrying Tmubl shRNA. Next, we designed and constructed a lentiviral vector carrying Tmub1 shRNA in order to to stably knockdown Tmub1 expression in rat liver BRL-3A cells. After infecting BRL-3A cells with four different Tmub1 vectors for $72 \mathrm{~h}$, we found that the most effective interferential sequence was 5'-GGTCTCAACACATACGACTGA-3'. Stably infected Tmub1 cells were sorted with a flow cytometer, expanded, and named BRL-3A/256 cells. Negative control cells were infected with the control vector, and named BRL-3A/Neg cells. Semiquantitative RT-PCR and western blotting assays showed that expression of Tmub1 mRNA and protein were significantly knocked down by one of the Tmub1 shRNA vectors in rat liver cells (Fig. 3). After the stably infected cells were treated with $15 \mathrm{ng} / \mathrm{ml}$ IL-6 for $12 \mathrm{~h}$, Tmub1 shRNA significantly blocked IL-6-induced expression of Tmub1 mRNA and protein (Fig. 3).

Effect of Tmubl knockdown on the proliferation of rat hepatocytes. We assessed the effect of Tmun 1 knockdown on hepatocyte proliferation in the presence or absence of IL-6 treatment. In the absence of IL- 6 treatment, $\left[{ }^{3} \mathrm{H}\right]$ thymidine incorporation into BRL-3A/256 cells was significantly higher than in control cells, but differences were more significant after IL-6 treatment (Fig. 4), indicating that Tmub1 knockdown synergized with IL-6 in inducing hepatocyte proliferation. The data demonstrate that Tmub1 functions as a negative regulator of cell proliferation.

Effect of Tmubl knockdown on influx of calcium ions in rat hepatocytes. Next, we determined the effect of Tmub1 knockdown on the influx of calcium ions in rat hepatocytes. After treatment with IL-6, fluorescence intensity of $\left[\mathrm{Ca}^{2+}\right]$ i quickly increased, and peaked at $40 \mathrm{sec}$ in all cells. However, the crest value of $\left[\mathrm{Ca}^{2+}\right] \mathrm{i}$ concentration was highest in BRL-3A/256 cells (Fig. 5), suggesting that Tmub1 knockdown has the ability to regulate $\mathrm{Ca}^{2+}$ influx in rat liver cells. 


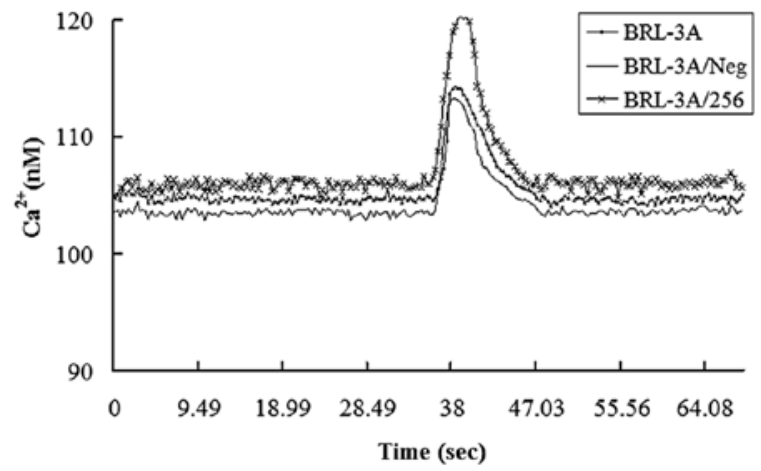

Figure 5. Effect of Tmub1 knockdown on the regulation of $\left[\mathrm{Ca}^{2+}\right]$ i. Parental, negative control, and Tmub1 knockdown cells were grown and treated with IL-6, and $\left[\mathrm{Ca}^{2+}\right] \mathrm{i}$ was measured. Crest value of fluorescence intensity in BRL-3A/256 cells was higher than in BRL-3A and BRL-3A/Neg cells $(\mathrm{P}<0.05)$, while there were no obvious differences between BRL-3A BRL-3A/Neg cells ( $\mathrm{P}>0.05)$.

Interaction of Tmubl with CAML protein. To explore Tmub1 signaling in the regulation of hepatocyte proliferation, we utilized laser confocal microscopy to examine colocalization of Tmub1 and CAML proteins in the cells. We then performed immunoprecipitation experiments followed by western blot- ting assays to assess Tmub1 interaction with CAML protein. The confocal microscopy images showed colocalization of Tmub1 with CAML protein in the cytoplasm of BRL-3A cells (Fig. 6). As shown in Fig. 6C1, green fluorescence is lighter than in the other two groups, which results in a partially red overlapping effect (Fig. 6C3), because Tmub1 expression is reduced. Moreover, results from our immunoprecipitation assays showed binding between Tmub1 and CAML proteins (Fig. 7). Furthermore, we also found that expression of CAML protein was upregulated after Tmubl knockdown, compared to the parental and negative control cells (Fig. 8).

\section{Discussion}

In this study, we investigated the role of Tmub1 in the regulation of rat hepatocyte proliferation induced by IL-6, and the potential underlying molecular events. Our data showed that expression of Tmub1 was upregulated by IL-6, proliferation of rat liver cells was enhanced by inhibition of Tmubl expression, and Tmub1 interacted with CAML in hepatocytes. Together, the data demonstrate that Tmub1 plays a negative role in regulating rat hepatocyte proliferation induced by IL-6 through interaction with CAML.
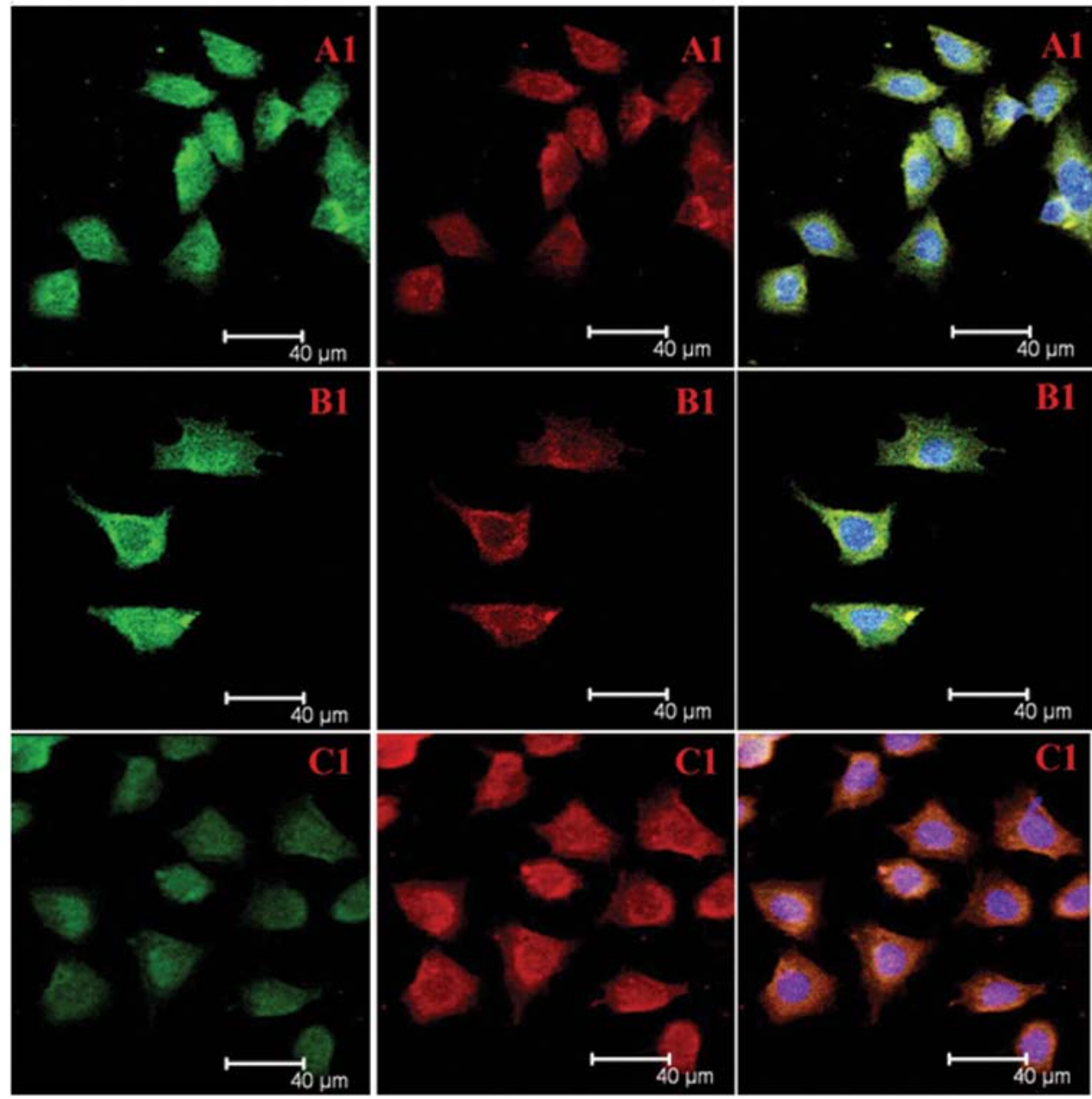

Figure 6. Colocalization of Tmub1 and CAML protein in the cytoplasm of hepatocytes. (A) Parental, (B) negative control, and (C) Tmub1 knockdown BRL-3A cells were grown and immunostained with anti-Tmub1 and CAML antibodies, and then viewed under a laser scanning confocal microscope. Green fluorescence shows Tmub1 and red shows CAML staining. The yellow fluorescence is overlapping Tmub1 and CAML, and the blue color indicates nuclear staining with 4,6-diamidino-2-phenylindole. 


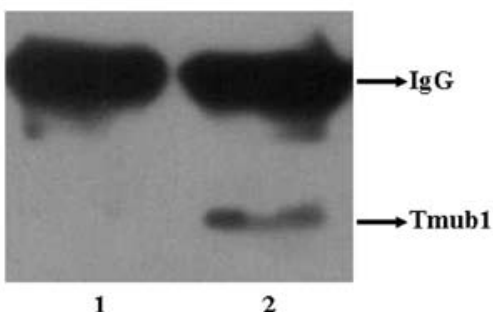

Figure 7. Immunoprecipitation-western blot analysis. BRL-3A cells were grown and subjected to immunoprecipitation and western blotting. Lane 1, negative control (immunoprecipitation with $\mathrm{IgG}$ and western blotting with anti-Tmub1 antibody), and Lane 2, CAML antibody (immunoprecipitation with anti-CAML antibody and western blotting with anti-Tmub1 antibody.

Our current data confirms that IL-6 treatment induces hepatocyte proliferation, and also significantly induces Tmub1 expression in rat hepatocytes. Previous studies revealed that IL-6 is extremely important in promoting cell proliferation and in directly or indirectly regulating the expression of various cell proliferation-related proteins during liver regeneration (12-14). However, the underlying molecular mechanisms remain unknown. A previous study demonstrated that IL-6 is able to activate STAT3 via the JAK pathway, and showed that activated STAT3, in turn, promotes expression of cell growthrelated genes, such as c-myc and AP-1, which induce G0/G1 cell cycle transition in hepatocytes (13).

However, our current study showed that knockdown of Tmub1 expression increased $\left[{ }^{3} \mathrm{H}\right]$ thymidine incorporation in rat hepatocytes, indicating that Tmub1 had the ability to inhibit proliferation of the liver cells. Moreover, Tmub1 shRNA synergized with IL- 6 in promoting rat hepatocyte proliferation. These data clearly demonstrate that Tmub1 is a negative player in hepatocyte proliferation or regeneration, and supports a previous study that demonstrated that overexpression of Tmub1 inhibits proliferation of hepatoma H-35 and NIH-3T3 cells (1). However, that study did not show the association between liver cell proliferation and knockdown of Tmub1 expression. Thus, it is unclear why IL-6 treatment and partial hepatectomy promote Tmub1 expression. These studies suggest that hepatocyte proliferation is very tightly controlled by both positive and negative regulators to ensure proliferation of adequate numbers of cells. In other words, differential gene expression maintains normal homeostasis in the human body.

Furthermore, our data showed that knockdown of Tmub1 expression in rat hepatocytes induced the expression of CAML proteins, which further confirmed the role of Tmub1 in the regulation of liver cell proliferation. These data are novel and provide insight into the function of Tmub1 in hepatocytes. In addition, we also found that the Tmub1 protein interacts and binds to CAML, which confirms a previous study showing an interaction between Tmub1 and CAML in the mouse brain (11). Morphologically, Tmub1 and CAML proteins were colocalized in the hepatocellular cytoplasm, and Tmub1 knockdown increased $\mathrm{Ca}^{2+}$ influx into hepatocytes. Early studies on CAML showed that CAML function is related to $\mathrm{Ca}^{2+}$ regulation. CAML affected the interaction of transcription factors with their target gene by regulating $\mathrm{Ca}^{2+}$ influx $(3,5)$. Our data suggest that interaction of Tmubl with CAML in hepatocytes affects $\mathrm{Ca}^{2+}$ influx to regulate the growth of liver cells.

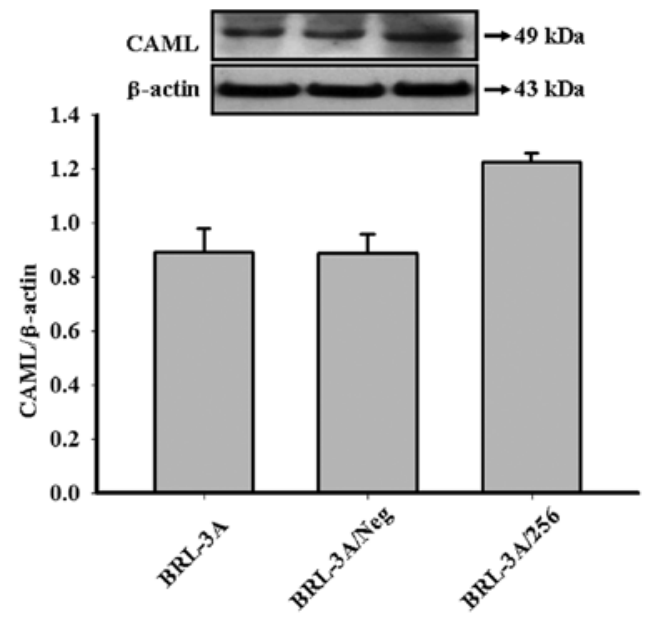

Figure 8. Effect of Tmub1 shRNA on the regulation of CAML expression Parental, negative control, and Tmubl knockdown cells were grown and subjected to western blot analysis of CAML expression in the presence of IL-6 treatment. Increased expression of CAML was statistically significant in BRL-3A/256 cells $(\mathrm{P}<0.05)$, but was not significant in BRL-3A or BRL-3A/ Neg cells $(P>0.05)$. The data from three independent experiments.

Increased expression of CAML, followed by Tmub1 knockdown, suggests that Tmub1 may participate in the degradation of CAML protein. Tmub1 protein contains a ubiquitin-like domain (ULD), which may participate in the degradation of other proteins that it interacts with. Although our understanding of ULD function is not unified, the key role played by ULDs in protein function is well-recognized (15). For example, some ULD proteins are involved in the degradation of targeting proteins by interacting with ubiqutin ligase E3 or recruiting a polyubiquitinated substrate to proteasomes (16-18). ULD proteins may promote or block this action according to different substrates (19). Some ULD proteins do not participate in the process of polyubiquitinated modification, but ULD is indispensable for the interaction with targeting proteins. For example, ULD is the key domain in I $\kappa$ B kinase $\beta$, which catalyzes NF- $\kappa$ B activation and regulates the subcellular localization of Homer3 (20,21). A recent study showed that Tmubl is involved in the degradation of the targeting protein by regulation of the ubiquitin-proteasome system that forms a complex with gp78 (22). However, further studies are needed to determine how Tmub1 regulates CAML expression.

In conclusion, although expression of Tmub1 was upregulated by IL- 6 treatment of rat hepatocytes, which is associated with hepatocyte proliferation, our current data demonstrates that Tmub1 is a negative regulator of hepatocyte growth (regeneration). Tmub1 knockdown promoted hepatocyte growth, expression of CAML, and $\mathrm{Ca}^{2+}$ influx into cells. Tmub1 was bound to CAML, and affected CAML levels in the hepatocytes. Future studies are needed to determine the role of Tmubl in vivo.

\section{Acknowledgements}

This study was supported in part by a grant from the Natural Science Foundation of China (no. 30972895) and from the Natural Science Foundation of Chongqing (no. 2009BA5014). 


\section{References}

1. Della Fazia MA, Castelli M, Bartoli D, Pieroni S, Pettirossi V, Piobbico D, Viola-Magni M and Servillo G: HOPS: a novel cAMP-dependent shuttling protein involved in protein synthesis regulation. J Cell Sci 118: 3185-3194, 2005.

2. Ping C, Xiaoling D, Jin Z, Jiahong D, Jiming D and Lin Z: Hepatic sinusoidal endothelial cells promote hepatocyte proliferation early after partial hepatectomy in rats. Arch Med Res 37: 576-583, 2006.

3. Bram RJ and Crabtree GR: Calcium signalling in T cells stimulated by a cyclophilin B-binding protein. Nature 371: 355-358, 1994.

4. Lim JH, Kim TY, Kim WH and Park JW: CAML promotes prolactin-dependent proliferation of breast cancer cells by facilitating prolactin receptor signaling pathways. Breast Cancer Res Treat 130: 19-27, 2010.

5. Von Bulow GU and Bram RJ: NF-AT activation induced by a CAML-interacting member of the tumor necrosis factor receptor uperfamily. Science 278: 138-141, 1997.

6. Tran DD, Russell HR, Sutor SL, van Deursen J and Bram RJ: CAML is required for efficient EGF receptor recycling. Dev Cell 5: 245-256, 2003.

7. Kumar R, Lutz W, Frank E and Im HJ: Immediate early gene $\mathrm{X}-1$ interacts with proteins that modulate apoptosis. Biochem Biophys Res Commun 323: 1293-1298, 2004.

8. Guo S, Lopez-Ilasaca M and Dzau VJ: Identification of calciummodulating cyclophilin ligand (CAML) as transducer of angiotensin II-mediated nuclear factor of activated T cells (NFAT) activation. J Biol Chem 280: 12536-12541, 2005.

9. Liu Y, Malureanu L, Jeganathan KB, Tran DD, Lindquist LD, van Deursen JM and Bram RJ: CAML loss causes anaphase failure and chromosome missegregation. Cell Cycle 8: 940-949, 2009.

10. Lagoudakis L, Garcin I, Julien B, Nahum K, Gomes DA, Combettes L, Nathanson MH and Tordjmann T: Cytosolic calcium regulates liver regeneration in the rat. Hepatology 52: 602-611, 2010.

11. Zhang W, Savelieva KV,Suwanichkul A, Small DL, Kirkpatrick LL, Xu N, Lanthorn TH and Ye GL: Transmembrane and ubiquitinlike domain containing 1 (Tmub1) regulates locomotor activity and wakefulness in mice and interacts with CAMLG. PLoS One 5: e11261, 2010.
12. Suh HN, Lee SH, Lee MY, Lee YJ, Lee JH and Han HJ: Role of interleukin-6 in the control of DNA synthesis of hepatocytes: involvement of PKC, p44/42 MAPKs, and PPARdelta. Cell Physiol Biochem 22: 673-684, 2008.

13. Streetz KL, Luedde T, Manns MP and Trautwein C: Interleukin 6 and liver regeneration. Gut 47: 309-312, 2000.

14. Gotohda N, Iwagaki H, Ozaki M, Kinoshita T, Konishi M, Nakagohri T, Takahashi S, Saito S, Yagi T and Tanaka N: IL-6 promotes compensatory liver regeneration in cirrhotic rat after partial hepatectomy. Cytokine 42: 372-378, 2008.

15. Hochstrasser M: Origin and function of ubiquitin-like proteins. Nature 458: 422-429, 2009.

16. Kny M, Standera S, Hartmann-Petersen R, Kloetzel PM and Seeger M: Herp regulates Hrd1-mediated ubiquitylation in a ubiquitin-like domain-dependent manner. J Biol Chem 286: 5151-5156, 2011.

17. Wilkinson CR, Seeger M, Hartmann-Petersen R, Stone M, Wallace M, Semple C and Gordon C: Proteins containing the UBA domain are able to bind to multi-ubiquitin chains. Nat Cell Biol 3: 939-943, 2001.

18. Schauber C, Chen L, Tongaonkar P, Vega I, Lambertson D, Potts W and Madura K: Rad23 links DNA repair to the ubiquitin/ proteasome pathway. Nature 391: 715-718, 1998.

19. Su V and Lau AF: Ubiquitin-like and ubiquitin-associated domain proteins: significance in proteasomal degradation. Cell Mol Life Sci 66: 2819-2833, 2009.

20. May MJ, Larsen SE, Shim JH, Madge LA and Ghosh S: A novel ubiquitin-like domain in I $\mathrm{B}$ kinase $\beta$ is required for functional activity of the kinase. J Biol Chem 279: 45528-45539, 2004.

21. Yatherajam G, Banerjee PP, McCorkell KA, Solt LA, Hanson EP, Madge LA, Kang S, Worley PF, Orange JS and May MJ: Cutting edge: association with I kappa B kinase beta regulates the subcellular localization of Homer3. J Immunol 185: 2665-2669, 2010.

22. Jo Y, Sguigna PV and DeBose-Boyd RA: Membrane-associated ubiquitin ligase complex containing gp78 mediates sterol-accelerated degradation of 3-hydroxy-3-methylglutaryl coenzyme a reductase. J Biol Chem 286: 15022-15031, 2011. 